\title{
Estimación de la Biomasa y Carbono Almacenado en la Cobertura Arbórea de la Región del Carare - Opón (Santander)
}

\section{Biomass and Stored Carbon Estimation in the Tree Cover of the Carare - Opón Region (Santander, Colombia).}

DOI: http:// http://dx.doi.org/10.17981/ingecuc.13.2.2017.09

Artículo de investigación científica. Fecha de recepción: 21 de agosto de 2017. Fecha de aceptación: 10 de octubre de 2017.

\author{
Mónica Lorena Barón Parra \\ Universidad Distrital Francisco José de Caldas. Bogotá (Colombia). \\ monica.baron26@gmail.com \\ Max Alejandro Triana Gómez \\ Universidad Distrital Francisco José de Caldas. Bogotá (Colombia). \\ mtriana@udistrital.edu.co
}

Para citar este artículo:

M. Barón Parra y M. Triana Gómez, "Estimación de la Biomasa y Carbono Almacenado en la Cobertura Arbórea de la Región del CarareOpón (Santander)," INGE CUC, vol. 13, no. 2, pp. 84-94, 2017. DOI: http://doi.org/10.17981/ingecuc.13.2.2017.09

\begin{abstract}
Resumen
Introducción- La causa principal del calentamiento global se debe al aumento de los gases de efecto invernadero que se han producido desde la revolución industrial. Se conoce que el dióxido de carbono (CO2) es el segundo gas que más aporta a esta problemática y que los bosques actúan como depósitos de carbono. De esta forma, los programas de reducción de emisiones por deforestación y degradación (REDD+) son un mecanismo de mitigación y adaptación al cambio climático; uno de sus propósitos es estimar la captura del carbono forestal.

Objetivo- Estimar el contenido de biomasa aérea y de carbono almacenado en el ecosistema y, así mismo, en las diez especies con mayor índice de valor de importancia (IVI), conocer cuál es la especie con mayor reserva de carbono y conocer si existen diferencias significativas en las variables estructurales de las especies.

Metodología- Las estimaciones de biomasa se realizaron mediante el método indirecto utilizando nueve ecuaciones alométricas que realizan esta estimación en función del diámetro a la altura del pecho $(\geq 10 \mathrm{~cm}$ a $1.30 \mathrm{~m})$ y la altura total. El carbono almacenado se calculó tomando el $50 \%$ de la biomasa estimada.

Resultados- Para el ecosistema se estimaron 465,41 t/0.05 ha de biomasa aérea y 232,70 t/0.05 ha de carbono almacenado. La especie con el valor más alto de biomasa y carbono fue Anacardium excelsum.

Conclusiones- Las diez especies con mayor IVI representan el $93,72 \%$ de la biomasa total del ecosistema; la más importante representa el 18,23\% estando relacionado con las variables estructurales de la vegetación como el área basal y la altura total.

Palabras clave- Calentamiento global; carbono forestal; ecuaciones alométricas; IVI; variables estructurales
\end{abstract}

Abstract

Introduction- The main cause of global warming is the increase of greenhouse gases since the industrial revolution; taking into account that carbon dioxide (CO2) is the second bigger contributor to this problem; and that the forests act as reservoirs of carbon. The reduction of emissions by degradation programs (REDD +) are a mechanism of mitigation and adaptation to climate change, one of their objectives is to estimate the capture of forest carbon.

Objective- This article aims to estimate the content of aerial biomass and stored carbon in the ecosystem, as well as in the ten species with the highest importance value index (IVI). This in order to know what is the species with the largest reserve of carbon and see whether there are differences in the structural variables of the species.

Methodology-The biomass estimations were made using an indirect method with nine allometric equations that estimate according to the diameter at breast height $(10 \mathrm{~cm}$ to $1.30 \mathrm{~m})$ and the total height. The carbon stored is calculated by taking the $50 \%$ of the estimated biomass.

Results-A value of $465.41 \mathrm{t} / 0.05$ ha of aerial biomass and $232.70 \mathrm{t} / 0.05$ ha of carbon stored were estimated for the ecosystem. The species with the highest value of biomass and carbon was Anacardium Excelsum.

Conclusions- The ten species with the highest IVI represent $93.72 \%$ of the total biomass of the ecosystem, the most important represents the $18.23 \%$, is related to the structural variables of the vegetation such as the basal area and the total height.

Keywords- Global warming; biomass; forest carbon; Allometric equations; IVI; Structural variables 


\section{INTRODUCCIÓN}

Actualmente el cambio climático es uno de los problemas ambientales más importantes. Se reconoce que su génesis se debe a la intervención antrópica generadora de los gases de efecto invernadero (GEI) [1], cuyas consecuencias han sido enormes por pérdidas en bienes materiales, biodiversidad y de la misma vida humana [2]. El dióxido de carbono $\left(\mathrm{CO}_{2}\right)$ es el segundo gas de mayor emisión a la atmósfera debido al cambio en el uso del suelo, principalmente en las zonas tropicales [3].

Este calentamiento global no solo afecta a los países industrializados sino a todo el planeta debido a que un impacto ambiental no repercute en el sitio donde ocurrió sino que repercute en toda la atmósfera del planeta [4].

Colombia, siendo un país en desarrollo, no es un gran generador de los GEI, pero sí contribuye en el cambio climático debido al aumento considerable de la deforestación que genera la pérdida de miles de hectáreas de bosques productores de oxígeno en el planeta. Esto causa la liberación de 6000 ton/año de $\mathrm{CO}_{2}$ [5] y se estima que la pérdida de cobertura forestal representa un $20 \%$ de la liberación de $\mathrm{CO}_{2}$ [6].

El ciclo del carbono es un concepto conocido en los sistemas naturales durante mucho tiempo, pero sólo hasta el año 1976 se mencionó por primera vez que los bosques son sumideros de carbono [7], ya que mediante el proceso de fotosíntesis tienen la capacidad de transformar el $\mathrm{CO}_{2}$ atmosférico en biomasa viva [8] [9] [10]. Sabiendo que la cobertura forestal es el principal depósito de carbono [11], se cuentan como estrategia de mitigación al cambio climático a nivel global [12] [13]this study describes the structural characteristics and carbon stocks of six natural forest types. This paper has a dual scope: i [14].

Un mecanismo de mitigación y adaptación al cambio climático son los proyectos de Reducción de Emisiones por Deforestación y Degradación de los Bosques (REDD+) siendo opciones político-efectivas. Entre sus fines está estimar las reservas de carbono almacenadas en los bosques [15] [16] [17], pues existen incertidumbres en cuanto a la disposición de las reservas de carbono debido a los cambios en el uso del suelo y la falta de conocimiento de los rebrotes [18] the BIOMASS mission was proposed to the European Space Agency for the third cycle of Earth Explorer Core missions and was selected for Feasibility Study (Phase A; por ejemplo, alrededor del 80\% del carbono almacenado en la superficie de la tierra se encuentra en los bosques tropicales [19].

Aunque los ecosistemas forestales son estrategias para mitigar el cambio climático existe poca información sobre sus reservas de carbono. El objetivo de este trabajo fue cuantificar la biomasa y carbono almacenado en este ecosistema y en las diez especies con mayor IVI, y, así mismo, conocer cuál es la especie que mayor carbono almacena.

\section{Materiales y Métodos}

\section{A. Área de estudio}

La región Carare-Opón se encuentra ubicada en el departamento de Santander, en el noreste de Colombia (Fig.1). Entre sus principales afluentes se encuentran los ríos Carare, al oeste, y Opón, al este [20]. Esta región forestal se caracteriza por presentar una zona de vida de bosque húmedo tropical (bhT), según Holdridge [21], una precipitación media anual de $2000 \mathrm{~mm}$ y la temperatura promedio varía entre $24^{\circ}$ y $30^{\circ} \mathrm{C}$.

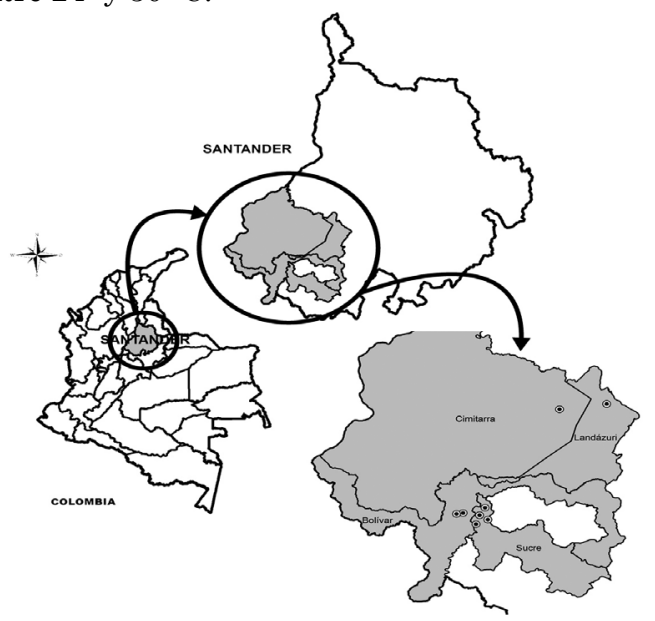

Fig. 1. Localización de la zona de estudio. Fuente: Autores.

\section{B. Trabajo de campo}

Se realizó un muestreo sistemático de parcelas ubicadas sobre líneas con una intensidad de $3 \%$ y una probabilidad del 95\%. Fueron establecidas 12 parcelas de 0.05 ha; luego se midieron todos los árboles con DAP (Diámetro a la Altura del Pecho) $\geq 10 \mathrm{~cm}$ a $1.30 \mathrm{~m}$, la altura comercial y la altura total, y la identificación de las especies se realizó mediante información primaria. El número total de individuos registrados en este inventario fue de 3821 .

\section{Estimación de la biomasa y el carbono}

La estimación de biomasa se realizó por medio del método indirecto: se tomaron 9 modelos alométricos buscados en la literatura, específicamente de la zona de vida de bosques húmedos (Tabla 1) debido a que no existe este tipo en la región. Para la selección de los modelos se tuvo en cuenta que la biomasa aérea estuviera en función del DAP y la altura total, siendo datos que siempre se obtienen en los inventarios.

El carbono almacenado corresponde al 50\% de la biomasa aérea [19]. 
Tabla 1. Modelos alométricos empleados para la estimación de la biomasa aÉrea de Árboles con DAP $\geq 10$ CM.

\begin{tabular}{|c|c|c|c|}
\hline $\mathrm{N}^{\circ}$ & Modelo & Región, zona de vida & Referencia \\
\hline 1 & $\mathrm{Ba}=\operatorname{EXP}(-2.1801+2.5624) * \operatorname{Ln}(\mathrm{DAP})$ & \multirow{2}{*}{ Ecuación general para bh-T } & \multirow{2}{*}[22]{} \\
\hline 2 & $\mathrm{Ba}=\operatorname{EXP}\left(-3.2249+(0.9885) * \ln \left(D A P^{2}\right) * \mathrm{Ht}\right)$ & & \\
\hline 3 & $\mathrm{Ba}=\mathrm{EXP}(-1.897+2.309) * \ln (\mathrm{DAP})$ & \multirow{2}{*}{ Costa Rica, bh-M } & \multirow{2}{*}{ [6] } \\
\hline 4 & $\mathrm{Ba}=\mathrm{EXP}(-2.647)+2.104 * \ln (\mathrm{DAP})+0.502 * \ln (H t)$ & & \\
\hline 5 & $\mathrm{Ba}=\operatorname{EXP}\left(0.33 * \ln (\mathrm{DAP})+\left(0.933 * \ln \left(\mathrm{DAP}^{2}\right)-0.122 * \ln \left(\mathrm{DAP}^{3}\right)-0.37\right)\right)$ & Brasil, bh-T & [23] \\
\hline 6 & $\mathrm{Ba}=13.2579-4.8945 *(\mathrm{DAP})+0.6713 *\left(\mathrm{DAP}^{2}\right)$ & \multirow{2}{*}{ Costa Rica, bh-T } & \multirow{2}{*}{ [24] } \\
\hline 7 & $\mathrm{Ba}=21.297022-(6.952649 *(D A P))+\left(0.7403 *\left(\mathrm{DAP}^{2}\right)\right)$ & & \\
\hline 8 & $\mathrm{Ba}=\operatorname{EXP}(-2.19+(2.54 * \ln (\mathrm{DAP})))$ & Neotrópico y Asia sudoriental, bh-T & {$[25]$} \\
\hline 9 & $\mathrm{Ba}=\mathrm{EXP}\left(-2.289+2.649 * \ln (\mathrm{DAP})-0.021 * \ln \left(\mathrm{DAP}^{2}\right)\right)$ & Vietnam, bh-MB & {$[26]$} \\
\hline
\end{tabular}

Convenciones: Ba (biomasa aérea), DAP (cm), Ht (m), bh-T (bosque húmedo tropical), bh-M (bosque húmedo montano), bh-MB (bosque húmedo montano bajo).

Fuente: Autores.

Tabla 2. Valores de correlación de Spearman utilizando los nueve modelos alométricos.

\begin{tabular}{|c|c|c|c|c|c|c|c|c|c|c|c|}
\hline Modelo & Ecosistema & $\begin{array}{c}\text { C. } \\
\text { brachypetala }\end{array}$ & $\begin{array}{c}\text { Inga } \\
\text { sp. }\end{array}$ & $\begin{array}{c}\text { V. } \\
\text { sebifera }\end{array}$ & $\begin{array}{c}\text { P. } \\
\text { laevis }\end{array}$ & $\begin{array}{c}\text { A. excels } \\
\text { um }\end{array}$ & $\begin{array}{c}\text { Lecythis } \\
\text { sp. }\end{array}$ & $\begin{array}{c}\text { H. } \\
\text { crepitans }\end{array}$ & $\begin{array}{c}\text { Brachycylix } \\
\text { vageleri }\end{array}$ & $\begin{array}{c}\text { Cecropia } \\
\text { sp. }\end{array}$ & $\begin{array}{c}\text { Xylopia } \\
\text { sp. }\end{array}$ \\
\hline $\mathbf{1}$ & 0,971 & 0,999 & 0,999 & 0,999 & 0,999 & 0,999 & 1,000 & 1,000 & 0,999 & 0,999 & 0,999 \\
\hline $\mathbf{2}$ & 0,998 & 0,986 & 0,984 & 0,992 & 0,984 & 0,989 & 0,991 & 0,985 & 0,987 & 0,984 & 0,988 \\
\hline $\mathbf{3}$ & 0,998 & 0,999 & 0,999 & 0,999 & 0,999 & 0,999 & 1,000 & 1,000 & 0,999 & 0,999 & 0,999 \\
\hline $\mathbf{4}$ & 0,996 & 0,998 & 0,996 & 0,998 & 0,996 & 0,995 & 0,997 & 0,994 & 0,996 & 0,995 & 0,997 \\
\hline $\mathbf{5}$ & 0,971 & 0,999 & 0,999 & 0,999 & 0,999 & 0,966 & 1,000 & 1,000 & 0,999 & 0,999 & 0,999 \\
\hline $\mathbf{6}$ & 0,994 & 0,999 & 0,999 & 0,999 & 0,999 & 0,999 & 1,000 & 1,000 & 0,999 & 0,999 & 0,999 \\
\hline $\mathbf{7}$ & 0,996 & 0,999 & 0,999 & 0,999 & 0,999 & 0,999 & 1,000 & 1,000 & 0,999 & 0,999 & 0,999 \\
\hline $\mathbf{8}$ & 0,971 & 0,999 & 0,999 & 0,999 & 0,999 & 0,999 & 1,000 & 1,000 & 0,999 & 0,999 & 0,999 \\
\hline $\mathbf{9}$ & 0,977 & 0,999 & 0,999 & 0,999 & 0,999 & 0,999 & 1,000 & 1,000 & 0,999 & 0,999 & 0,999 \\
\hline
\end{tabular}

Fuente: Autores

\section{Análisis de datos}

El procesamiento general de los datos se realizó con el programa Excel. Se calculó el Índice de Valor de Importancia (IVI) y con base en éste se definieron las diez especies de mayor peso ecológico (Anexo 1), así mismo, se estimó la biomasa aérea mediante ecuaciones alométricas.

Se utilizó el coeficiente de correlación de Spearman en la estimación de la biomasa para conocer el modelo que presenta la mayor correlación media comparado con los otros modelos. También se realizó un análisis de varianza mediante la prueba Kruskall-Wallis (no paramétrico) con el fin de establecer si existen diferencias significativas entre las variables: área basal, número de individuos, altura total, biomasa y carbono. Estas pruebas estadísticas se realizaron con el programa R Project 3.1.1.

\section{Resultados y Discusión}

\section{A. Estimación de la biomasa}

De acuerdo con los resultados (Tabla 2), los valores se encuentran entre 0,96 y 1 . Para seleccionar el modelo más ajustado se tuvieron en cuenta las condiciones climáticas similares a la zona de estudio; para las especies es el modelo 5 y para el ecosistema, el modelo 2.

En las distribuciones de biomasa por clase diamétrica, se tiene que para el ecosistema y las especies (Fig.2) las estimaciones más altas en el ecosistema se presentaron en los modelos 1 y 8 ; siendo modelos que utilizan el DAP como variable estimadora de la biomasa, que, según [22] [24] [27] [28], proporciona buenas estimaciones; las más bajas fueron en los modelos 4 y 3. En cuanto a las diez especies, la estimación de la biomasa fue alta con los modelos 5 (reportado por [29]) y 1. 

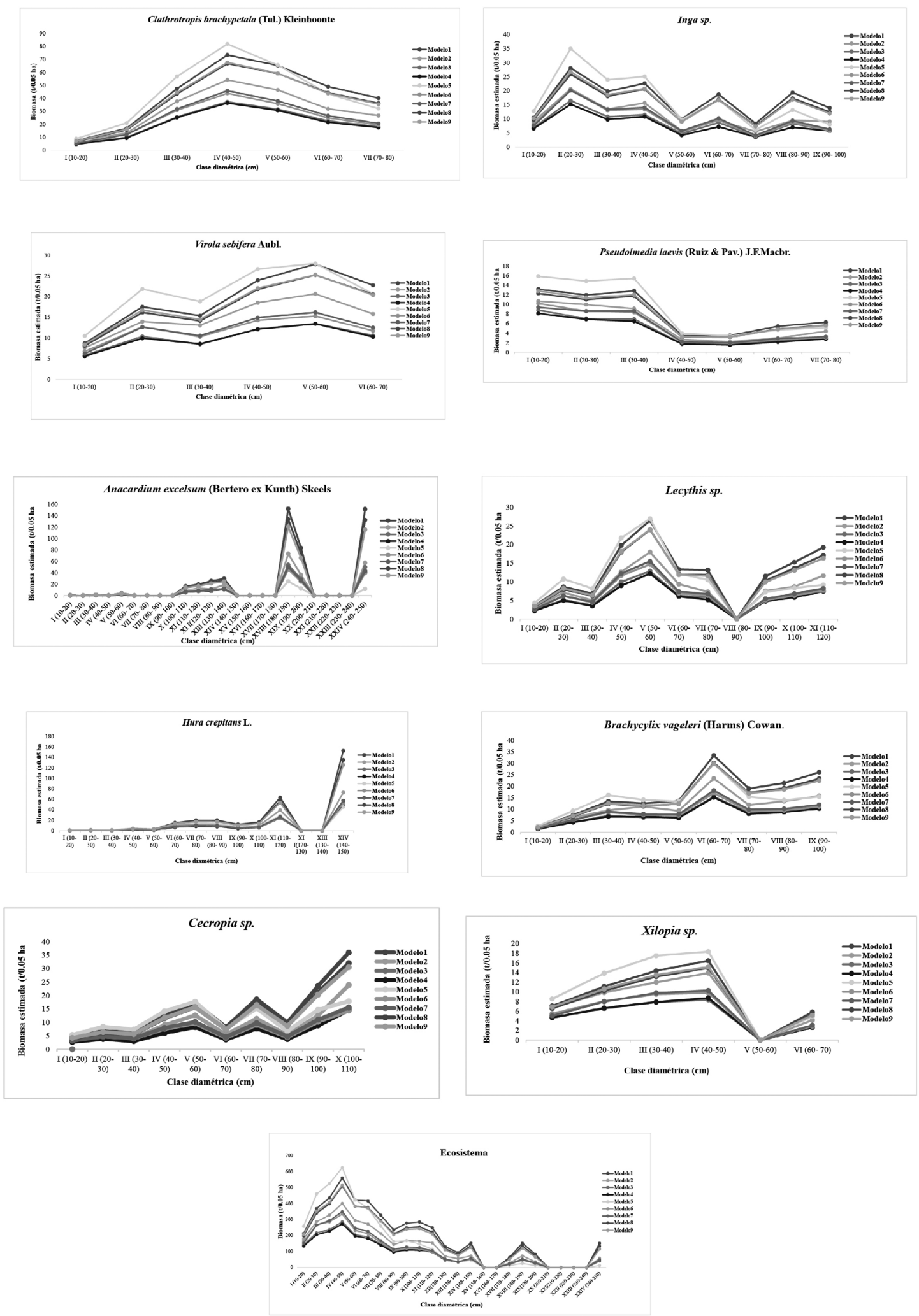

Fig. 2. Distribución de la biomasa aérea por clase diamétrica utilizando los 9 módelos alométricos, Fuente: Autores. 
Un modelo alométrico con más variables en función de la biomasa como el DAP, la altura total y la densidad de la madera genera una mayor fiabilidad [30] [31] [32] [33] [26], pero existe la posibilidad de presentar mayores errores debido a que hay incertidumbre en las mediciones [34]there is an urgent need of reliable estimates of biomass and carbon pools in tropical forests, most especially in Africa where there is a serious lack of data. Information on current annual increment (CAI [35]. Así mismo, el uso de ecuaciones específicas para cada especie genera valores más confiables que utilizando ecuaciones generales [36], pero se debe tener en cuenta que poder obtener resultados más precisos es más costoso.

\section{B. Variación de la biomasa y variables estructurales}

Entre las especies, la variación de la biomasa estuvo entre $25,75+/-0,20 \mathrm{t} / 0,05$ ha y 5,25 +/- 0,10 t/0,05 ha; con el carbono estimado fue $12,87 \mathrm{t} / 0,05$ ha hasta 2,62 t/0,05 ha; y para el área basal estuvo entre 2- 0,02 $\mathrm{m}^{2} / 0,05$ ha. Los valores altos se presentaron con la especie Clathrotropis brachypetala (Tul.) Kleinhoonte, y los bajos con Xylopia sp.

En cuanto a la altura total, los rangos de variación fueron de 25,53 m con $A$. excelsum hasta 12,74 $\mathrm{m}$ con $P$. laevis. En cuanto a la densidad (número de individuos), no se presentaron diferencias significativas. La variación de biomasa fue de $249,96+/-0,74$ t/0,05 ha con respecto al ecosistema, y del carbono fue de 124,98 t/0,05 ha (Tabla 3).

En la Fig. 3 la especie Inga sp. presentó la mayor mediana con 19 ind. /0,05 ha mientras que la menor fue $A$. excelsum con 1 ind. /0,05 ha.
Con respecto a la altura total, $A$. excelsum presenta la mediana más alta con $26 \mathrm{~m}$, teniendo un individuo con un valor mínimo de $10.5 \mathrm{~m}$; la más baja fue $12 \mathrm{~m}$ con la especie $P$. laevis.

Para el área basal, $A$. excelsum presenta el valor mayor con $1 \mathrm{~m}^{2} / 0,05$ ha y con una mediana baja de $0,2 \mathrm{~m}^{2} / 0,05$ ha se encuentran tres especies $(C$. brachypetala, Inga sp. y Virola sp.).

En cuanto a la biomasa se tiene alta variación en A. excelsum, presentando valores altos con la altura total y el área basal, y la menor variación se presentó con $P$. laevis teniendo valor bajo en la altura total.

\section{Variación de la biomasa y variables estructurales por clase diamétrica}

El anexo 2 muestra por clase diamétrica la distribución de las variables de la estructura de la vegetación (densidad, altura total, área basal), biomasa y carbono en el ecosistema y en las especies más importantes, donde los valores corresponden al promedio, a excepción de la altura total, que toman los valores máximos y mínimos. El ecosistema obtuvo 465,41 t/0,05 ha de biomasa aérea y 232,70 t/0,05 ha de carbono almacenado. Los valores altos se presentaron en las especies $A$. excelsum con 79,51 t/0,05 ha de biomasa y 39,75 t/0,05 ha de carbono y, en segundo lugar, se encuentra Hura crepitans con 73,87 t/0,05 ha y 36,93 t/0,05 ha, respectivamente, valores directamente relacionados con el área basal y la altura total.

Para las dos especies con valores altos de biomasa aérea y carbono almacenado, su mayor contenido se encuentra en las últimas clases diamétricas, lo que muestra que estas especies mantienen un alto número de individuos maduros, reportado por [33].

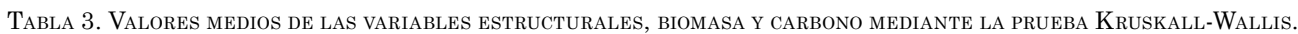

\begin{tabular}{|c|c|c|c|c|c|}
\hline Especie & $\begin{array}{c}\text { Densidad } \\
\text { (ind } / 0,05 \text { ha) }\end{array}$ & $\begin{array}{l}\text { Altura } \\
\text { total (m) }\end{array}$ & $\begin{array}{c}\text { ärea basal } \\
(\mathrm{m} 2 / 0,05 \mathrm{ha})\end{array}$ & $\begin{array}{l}\text { Biomasa } \\
(\mathrm{t} / 0,05 \mathrm{ha})\end{array}$ & $\begin{array}{l}\text { Carbono } \\
\text { (t/0,05 ha) }\end{array}$ \\
\hline Clathrotropis brachypetala (Tul.) Kleinhoonte & 18.41 & 17.06 & 2.00 & $25.75+/-0.20$ & 12.87 \\
\hline Inga sp. & 19.00 & 14.22 & 1.28 & $15.08+/-0.20$ & 7.54 \\
\hline Virola sebifera Aubl. & 14.08 & 16.54 & 0.86 & $10.52+/-0.16$ & 5.26 \\
\hline Pseudolmedia laevis (Ruiz \& Pav.) J.F. Macbr. & 15.18 & 12.74 & 0.53 & $5.74+/-0.09$ & 2.87 \\
\hline Anacardium excelsum (Bertero ex Kunth) Skeels & 1.85 & 25.53 & 2.57 & $13.29+/-0.19$ & 6.64 \\
\hline Lecythis sp. & 7.81 & 15.81 & 0.89 & $10.85+/-0.14$ & 5.42 \\
\hline Hura crepitans L. & 3.33 & 20.66 & 1.69 & $15.88+/-0.24$ & 7.94 \\
\hline Brachycylix vageleri (Harms) Cowan. & 7.3 & 18.02 & 1.05 & $13.05+/-0.23$ & 6.52 \\
\hline Ceccropia sp. & 7.7 & 16.14 & 0.97 & $11.47+/-0.18$ & 5.73 \\
\hline Xylopia sp. & 9.75 & 15.75 & 0.02 & $5.25+/-0.100$ & 2.62 \\
\hline Ecosistema & 318.41 & 14.86 & 27.24 & $249.96+/-9.74$ & 124.98 \\
\hline Estadístico H (10, N = 1143) & 341.346 & 162.29 & \multicolumn{3}{|c|}{176.73} \\
\hline Nivel de significancia (p) & $8.46 \mathrm{e}-05$ & $<2.2 \mathrm{e}-16$ & \multicolumn{3}{|c|}{$<2.2 \mathrm{e}-16$} \\
\hline
\end{tabular}

Fuente: Autores. 

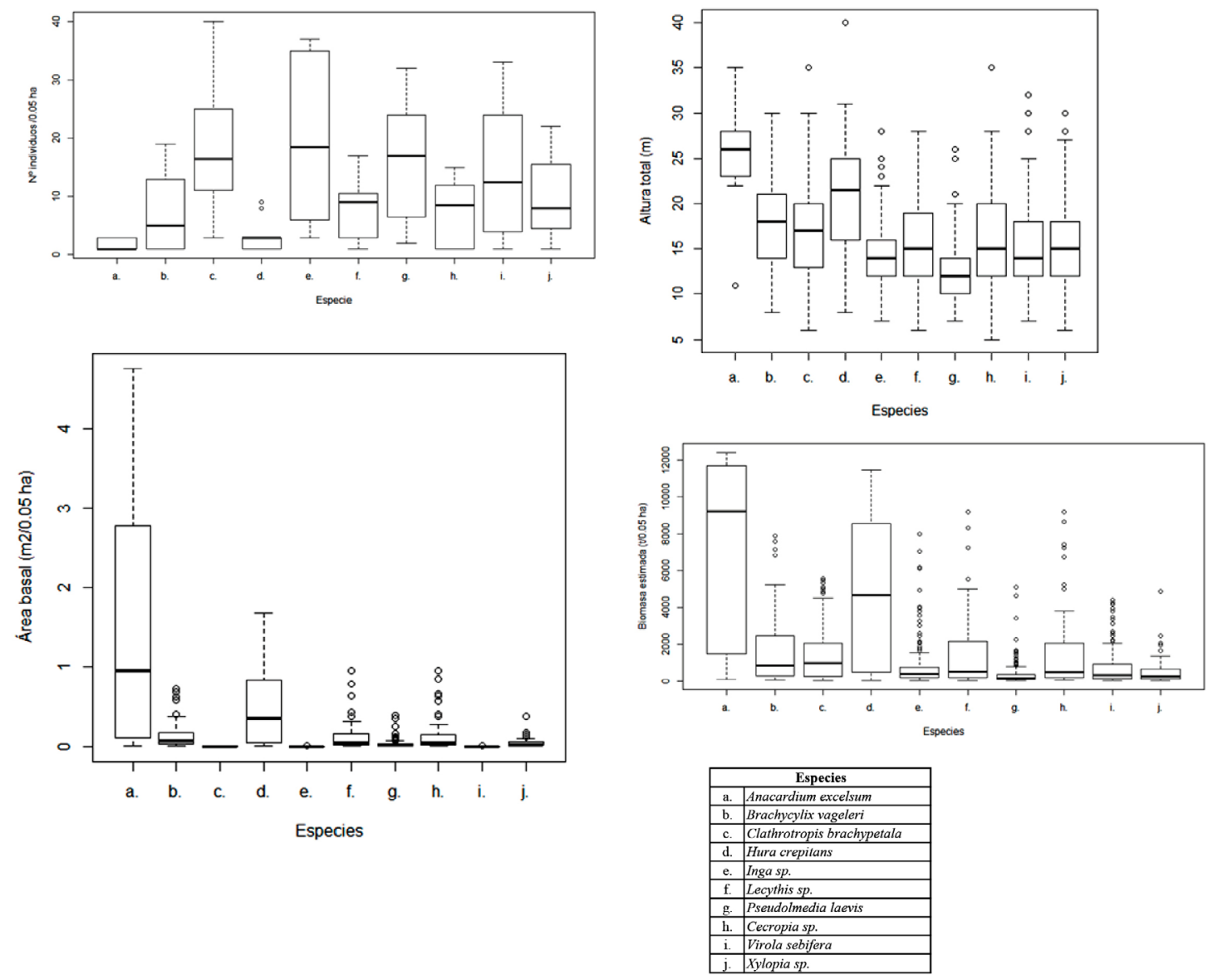

Fig. 3. Variación de parámetros estructurales y biomasa.

Fuente: Autores.

A nivel de ecosistema, los valores altos se encuentran en las primeras clases diamétricas, demostrando que ha existido intervención antrópica (Fig.4).

\section{Importancia de los bosques}

El cambio de uso del suelo de pastizal o cultivo agrícola por bosques es una medida efectiva para reducir los GEI [37] [38] encouraging countries to initiate actions to reduce emissions from deforestation and forest degradation in developing countries (REDD, así mismo, la presencia de éstos en sistemas agrícolas [39]. Coberturas arbóreas primarias degradadas por minería para obtener estimación de biomasa similar a la que tenía antes de la intervención requiere de mil años de recuperación [40]. Esto demuestra la importancia de preservar y conservar los bosques que son fuente de vida para mantener la biodiversidad de la flora y fauna, y también brindar los servicios ecosistémicos, como la captura del carbono.
Se ha demostrado que el uso de los desperdicios forestales (ramas, copas de árboles, tocones) y agrícolas para la generación de energía proporciona menores cantidades de emisión que el uso de combustibles fósiles [18], [41]-[43] con una disminución de entre el $11 \%$ y el $15 \%$ [44].

\section{Conclusiones}

Se pone en evidencia que los valores de biomasa corresponden a las especies de mayor índice de importancia; las 10 especies mejor representadas soportan el 93,72\% de la biomasa total del ecosistema; así mismo, las dos especies con mayor cantidad de biomasa aérea son Anacardium excelsum (18,23\%) y Hura crepitans (16,24\%), condición típica de bosques intervenidos, donde especies de carácter heliófito durable poseen mayor peso ecológico. Esta posición dominante está relacionada con las variables estructurales de la vegetación como el área basal y la altura total. De igual forma, se pone en evidencia la misma situación con el carbono almacenado, ya que está en función de la biomasa. 

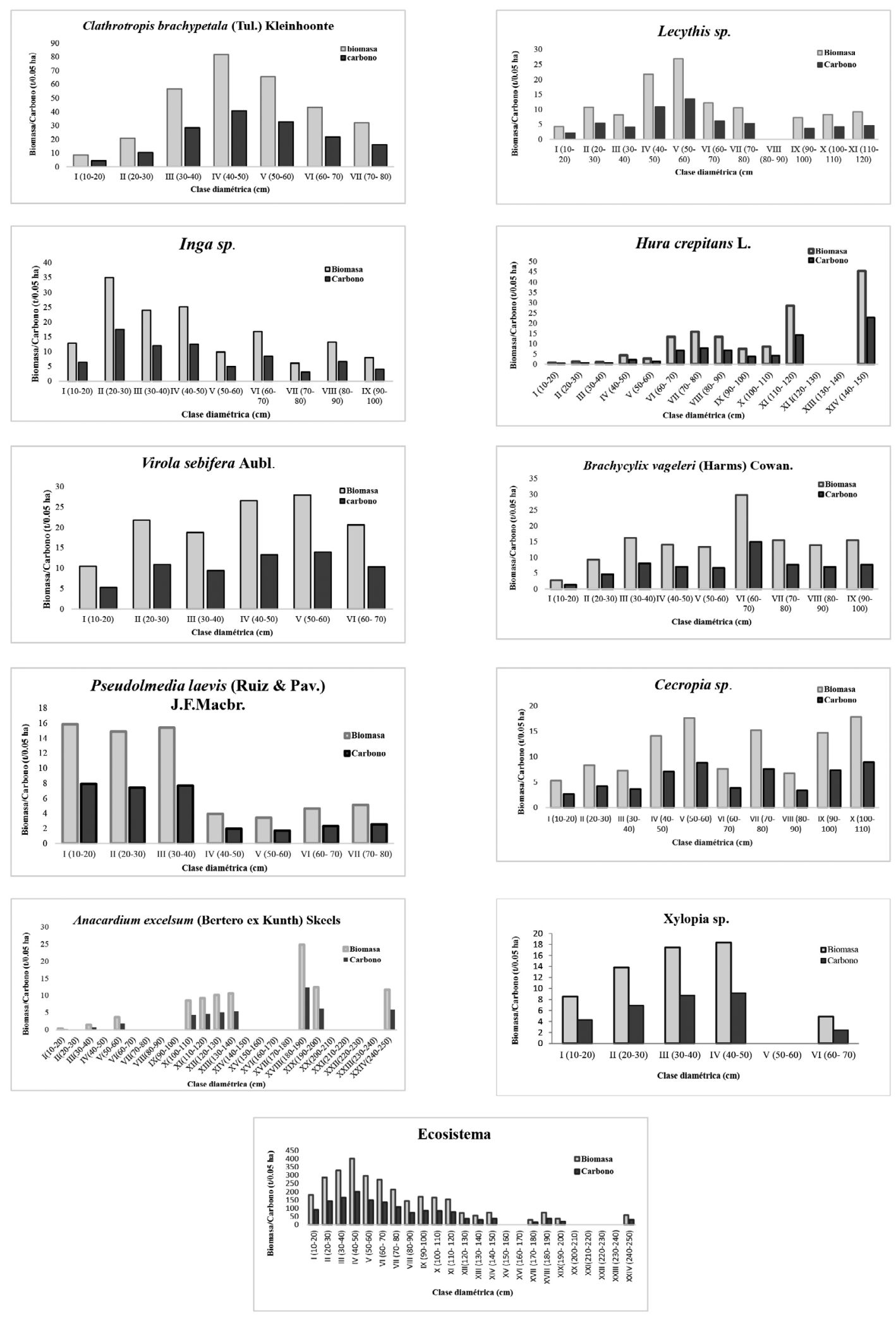

Fig. 4. Distribución de la biomasa aérea y carbono por clase diamétrica. Fuente: Autores. 
La utilización de los modelos mostró similitudes en cuanto a los valores de la biomasa estimada presentando valores más altos en aquellos que se calculan en función del DAP y valores más bajos cuando se combinan las variables DAP y la altura total. Esta evidencia permite inferir la robustez de la variable DAP, sin embargo, se requieren mayores investigaciones para descartar definitivamente variables tan importantes como la altura y la densidad de la madera. Entre tanto, los autores consideran que estas y otras variables (altura y la densidad de la madera, gremio ecológico, etc.) pueden proporcionar importante información relativa a los niveles de degradación de estos bosques, más allá de sus reservas de carbono.

Mediante el uso de las ecuaciones alométricas es posible tener una visión del potencial de captura de carbono en los bosques y, así mismo, poder utilizar los datos para la toma de decisiones con respecto a las medidas de mitigación y manejo en el contexto de la variabilidad climática.

\section{FINANCIACIÓN}

El presente es un artículo de investigación derivado del Proyecto para el desarrollo participativo de las actividades de reactivación del proyecto forestal Carare-Opón. Subproyecto de investigación: "Parcelas permanentes para la dinámica y silvicultura de especies forestales.” Proyecto financiado por la Universidad Distrital Francisco José de Caldas y la Corporación Desarrollo y Paz del Magdalena Medio. Fecha de inicio del subproyecto: junio de 2003. A la fecha se siguen monitoreando las parcelas permanentes.

\section{REFERENCIAS}

[1] F. Castro, "Cambio climático y protocolo de Kioto. Ciencia y Estrategias. Compromisos para España,” Rev. Esp Salud Pública, vol. 7, núm. 2, pp. 191-210, marzo-abril, 2005. [En línea]. Disponible en: http://www.redalyc.org/ pdf/170/17079209.pdf

[2] A. Yepes-Mayorga, "Cambio Climático: estrategias de gestión con el tiempo en contra...," Orinoquia, vol. 16, núm. 1, pp. 77-92, 2012. [En línea]. Disponible en: http:// www.scielo.org.co/pdf/rori/v16n1/v16n1a09.pdf

[3] J. A. Benjamín y O. Masera, "Captura de carbono ante el cambio climático," Madera y Bosques, vol. 7, núm. 1, pp. 3-12, 2001. [En línea]. Disponible en: http://www.redalyc.org/pdf/617/61770102.pdf

[4] F. Estenssoro, "Crisis ambiental y cambio climático en la política global: Un tema crecientemente complejo para América Latina," Universum, vol. 2, núm. 25, pp 57-77, julio, 2010. [En línea]. Disponible en: https://doi. org/10.4067/S0718-23762010000200005

[5] FAO, "Los bosques y el cambio climático. La gestión forestal es fundamental para afrontar el cambio climático," 2006. [En línea]. Disponible en: http://www.fao.org/Newsroom/es/focus/2006/1000247/index.html

[6] B. Schlegel, "Estimación de la biomasa y carbono en bosques del tipo forestal siempre verde," Presentando en SIMMCCEF, Chile, octubre, 2001. [En línea]. Disponible en: https://www.uach.cl/procarbono/pdf/simposio_carbono/45_schlegel.PDF
[7] A. Vargas-Mena y A. Sandoval. (2004, Enero). "La captura de carbono en bosques: ¿una herramienta para la gestión ambiental?," Gac. Ecológica, vol. 1, núm. 70, pp. 5-18. [En línea]. Disponible en: http://www.redalyc.org/ pdf/539/53907001.pdf

[8] M. G. Pece, C. Gaillard, M. Galíndez y M. Acosta, "Bosques y forestaciones como sumideros de carbono en el parque chaqueño," Presentado en FCF-UNSE, 2000. [En línea]. Disponible en: http://fcf.unse.edu.ar/eventos/2jornadas-forestales/pdfs/Bosques $\% 20 \mathrm{y} \% 20$ forestaciones $\% 20$ como $\% 20$ sumideros $\% 20$ de $\% 20$ carbono $\% 20$ en $\% 20$ el\%20parque $\% 20$ chaqueno.pdf

[9] A. Vásquez y H. Arellano, "Estructura, Biomasa Aérea y Carbono Almacenado en los Bosques del Sur y Noroccidente de Córdoba," Colomb. Divers. Biótica XII. La región Caribe Colomb., pp. 963-1009, 2012 [En línea]. Disponible en: https://arxiv.org/ftp/arxiv/papers/1208/1208.0248.pdf

[10] A. M. Aldana, B. Villanueva, A. Cano, D. F. Correa, M. N. Umaña, L. F. Casas, S. Cárdenas, L. F. Henao-Díaz y P. R. Stevenson (2017) "Drivers of biomass stocks in Northwestern South American forests: Contributing new information on the Neotropics". Forest Ecology and Management, vol. 389, pp. 86-95, 2017. [En línea]. Disponible en: http://dx.doi.org/10.1016/j.foreco.2016.12.023

[11] Banco Mundial, "Por qué los bosques son fundamentales para el clima, el agua, la salud y los medios de subsistencia," marzo 2016. [En línea]. Disponible en: http://www. bancomundial.org/es/news/feature/2016/03/18/whyforests-are-key-to-climate-water-health-and-livelihoods

[12] FAO, "La gestión de los bosques ante el cambio climático," 2010. [En línea]. Disponible en: http://www.fao.org/ docrep/014/i1960s/i1960s00.pdf

[13] E. Mattsson, M. Ostwald, G. Wallin y S. P. Nissanka, "Heterogeneity and assessment uncertainties in forest characteristics and biomass carbon stocks: Important considerations for climate mitigation policies," Land use policy, vol. 59, pp. 84-94, agosto 2016. [En línea]. Disponible en: http://www.sciencedirect.com/science/article/ pii/S0264837716308766

[14] C. R. Sanquetta, A. P. Dalla Corte y G. C. Benedet Maas, "El rol del bosque en el cambio climático," Quebracho (Santiago del Estero), vol. 19, núm. 2, pp. 84-96, diciembre 2011. [En línea]. Disponible en: http://www.sciencedirect.com/science/article/pii/S0264837716308766

[15] S. W. Chou y E. E. Gutierrez-Espeleta, "Equation for estimating tree biomass in tropical forests of Costa Rica," Tecnología en marcha, vol. 26, núm. 2, pp. 41-54, septiembre 2012. [En línea]. Disponible en: https://dialnet. unirioja.es/descarga/articulo/4835699.pdf

[16] C. B. Field et. al. "Cambio climático 2014 Impactos, adaptación y vulnerabilidad." 2014. [En línea]. https:// www.ipcc.ch/pdf/assessment-report/ar5/wg2/ar5_wgII_ spm_es.pdf

[17] J. F. Phillips et al., "Estimación de las reservas actuales (2010) de carbono almacenadas en la biomasa aérea en bosques naturales de Colombia," octubre, 2010. [En línea]. Disponible en: http://documentacion.ideam.gov. co/openbiblio/bvirtual/022100/EstimaciondelasReservas2010.pdf

[18] T. Le Toan et al., "The BIOMASS mission: Mapping global forest biomass to better understand the terrestrial carbon cycle," Remote Sens. Environ., vol. 115, núm. 11, pp. 2850-2860, 2011. [En línea]. Disponible en: https:// doi.org/10.1016/j.rse.2011.03.020

[19] A. Yepes-Quintero, A., Duque-Montoya A. J., Navarrete-Encinales D., Phillips-Bernal, J., CabreraMontenegro, E., Corrales-Osorio et al., "Estimación de las Reservas y Pérdidas de Carbono por Deforestación en los Bosques del Departamento de Antioquia, Colombia," Actual. Biológicas, vol. 33, núm. 95, pp. 193-208, 2011. [En línea]. Disponible en: http://www. scielo.org.co/scielo.php?script=sci_arttext\&pid=S0304$35842011000200005 \& \operatorname{lng}=\mathrm{es} \& \operatorname{tlng}=\mathrm{es}$ 


\section{ESTIMACIÓN DE LA BIOMASA Y CARBONO ALMACENADO EN LA COBERTURA \\ ARBÓREA DE LA REGIÓN DEL CARARE - OPÓN (SANTANDER).}

[20] R. Franco y J. M. Rodríguez, "Análisis multitemporal satelital de los bosques del Carare - Opón, mediante imágenes landsat de 1991 y 2002 ," Colomb. For., vol. 9, núm. 18, pp. 157-162, 2005. [En línea]. Disponible en: http:// revistas.udistrital.edu.co/ojs/index.php/colfor/article/ view/3054

[21] H. J. Gutiérrez Rey, "Aproximación a un modelo para la evaluación de la vulnerabilidad de las coberturas vegetales de Colombia ante un posible cambio climático utilizando Sistemas de Información Geografica SIG con énfasis en la vulnerabilidad de las coberturas nival y de páramo de Colombia," Páramos y Ecosistemas Alto Andin. Colomb. en Condición HotSpot Glob. Clim. Tensor, vol. 6, pp. 335-377, 2002. [En línea]. Disponible en: http:// documentacion.ideam.gov.co/openbiblio/bvirtual/000689/ Capitulo5.pdf

[22] A. N. Djomo, A. Ibrahima, J. Saborowski y G. Gravenhorst, "Allometric equations for biomass estimations in Cameroon and pan moist tropical equations including biomass data from Africa," For. Ecol. Manage, vol. 260, núm. 10, pp. 1873-1885, 2010. [En línea]. Disponible en: http://dx.doi.org/10.1016/j.foreco.2010.08.034

[23] H. Quinto-Mosquera y F. Moreno, "Dinámica de la Biomasa Aérea en un Bosque Pluvial Tropical del Chocó Biogeográfico," Rev. Fac. Nac. Agron. - Medellín, vol. 64, núm. 1, pp. 5917-5936, 2011. [En línea]. Disponible en: https://revistas.unal.edu.co/index.php/refame/article/ view/26399/37133

[24] M. Segura y M. Kanninen, "Allometric Models for Tree Volume and Total Aboveground Biomass in a Tropical Humid Forest in Costa Rica," Biotropic, vol. 37, núm. 1, pp. 2-8, 2005. [En línea]. Disponible en: https://doi. org/10.1111/j.1744-7429.2005.02027.x

[25] J. R. Me Chave, B. R. Ra y M.-A. Dubois, "Estimation of biomass in a neotropical forest of French Guiana: spatial and temporal variability," J. Trop. Ecol. vol. 17, pp. 79-96, 2011. [En línea]. Disponible en: https://doi. org/10.1017/S0266467401001055

[26] B. Huy, K. P. Poudel y H. Temesgen, "Aboveground biomass equations for evergreen broadleaf forests in South Central Coastal ecoregion of Viet Nam: Selection of ecoregional or pantropical models," For. Ecol. Manage., vol. 376, pp. 276-283, 2016. [En línea]. Disponible en: https:// doi.org/10.1017/S0266467401001055

[27] C. A. Sierra et al., "Total carbon stocks in a tropical forest landscape of the Porce region, Colombia," For. Ecol. Ma nage., vol. 243, núms. 2-3, pp. 299-309, 2007. [En línea]. Disponible en: http://www.sciencedirect.com/science/article/pii/S0378112707002411

[28] N. Chan, S. Takeda, R. Suzuki y S. Yamamoto, "Establishment of allometric models and estimation of biomass recovery of swidden cultivation fallows in mixed deciduous forests of the Bago Mountains, Myanmar," For. Ecol. Ma nage., vol. 304, pp. 427-436, 2013. [En línea]. Disponible en: http://dx.doi.org/10.1016/j.foreco.2013.05.038

[29] H. Quinto-Mosquera y F. Moreno, "Dinámica de la Biomasa Aérea en un Bosque Pluvial Tropical del Chocó Biogeográfico," Rev. Fac. Nac. Agron. - Medellín, vol. 64, núm. 1, pp. 5917-5936, 2011. [En línea]. Disponible en: https://revistas.unal.edu.co/index.php/refame/article/ view/26399/37133

[30] E. Álvarez et al., "Tree above-ground biomass allometries for carbon stocks estimation in the natural forests of $\mathrm{Co}$ lombia," For. Ecol. Manage., vol. 267, pp. 297-308, 2012. [En línea]. Disponible en: http://dx.doi.org/10.1016/j.foreco.2011.12.013

[31] S. M. Stas, E. Rutishauser, J. Chave, N. P. R. Anten y Y. Laumonier, "Estimating the aboveground biomass I, an old secondary forest on limestone in the Moluccas, Indonesia: Comparing locally developed versus existing allometric models," For. Ecol. Manage., vol. 389, pp. 27-34, 2017. [En línea]. Disponible en: http://dx.doi.org/10.1016/j. foreco.2016.12.010
[32] A. Ngomanda et al., "Site-specific versus pantropical allometric equations: Which option to estimate the biomass of a moist central African forest?," For. Ecol. Manage., vol. 312, pp. 1-9, 2014. [En línea]. Disponible en: http:// dx.doi.org/10.1016/j.foreco.2013.10.029

[33] A. M. Aldana et al., "Drivers of biomass stocks in Northwestern South American forests: Contributing new information on the Neotropics," For. Ecol. Manage., vol. 389, pp. 86-95, 2017. [En línea]. Disponible en: http:// dx.doi.org/10.1016/j.foreco.2016.12.023

[34] A. N. Djomo, A. Knohl y G. Gravenhorst, "Estimations of total ecosystem carbon pools distribution and carbon biomass current annual increment of a moist tropical forest," For. Ecol. Manage., vol. 261, núm. 8, pp. 1448-1459, 2011. [En línea]. Disponible en: http://dx.doi. org/10.1016/j.foreco.2011.01.031

[35] S. K. Behera, N. Sahu, A. K. Mishra, S. S. Bargali, M. D. Behera y R. Tuli, "Aboveground biomass and carbon stock assessment in Indian tropical deciduous forest and relationship with stand structural attributes," Ecol. Eng., vol. 99, pp. 513-524, 2017. [En línea]. Disponible en: http://dx.doi.org/10.1016/j.ecoleng.2016.11.046

[36] A. N. Djomo y C. D. Chimi, "Tree allometric equations for estimation of above, below and total biomass in a tropical moist forest: Case study with application to remote sensing," For. Ecol. Manage., vol. 391, pp. 184-193, 2017. [En línea]. Disponible en: http://dx.doi.org/10.1016/j.foreco.2017.02.022

[37] J. Pelletier, K. R. Kirby y C. Potvin, "Significance of carbon stock uncertainties on emission reductions from deforestation and forest degradation in developing countries," For. Policy Econ., vol. 24, pp. 3-11, 2012. [En línea]. Disponible en: https://doi.org/10.1016/j.forpol.2010.05.005

[38] S. G. de Godoi et al., "The conversion of grassland to acacia forest as an effective option for net reduction in greenhouse gas emissions," J. Environ. Manage., vol. 169, pp. 91-102, 2016. [En línea]. Disponible en: https://doi. org/10.1016/j.jenvman.2015.11.057

[39] M. Ibrahim y J. Mora, Potencialidades de los sistemas silvopastoriles para la generación de servicios ambientales: memorias de una conferencia electrónica realizada entre septiembre y diciembre del 2001, Turrialba, Costa Rica, 2006 [En línea]. Disponible en: http://www.flacsoandes.edu.ec/libros/124741-opac

[40] H. Quinto, J. Cuesta, I. J. Mosquera, L. Palacios y H. Peñaloza, "Biomasa vegetal en zonas degradadas por minería en un bosque pluvial tropical del Chocó Biogeográfico," Biodivers. Neotrop., vol. 3, núm. 1, pp. 53-64, 2013. [En línea]. Disponible en: http://dx.doi.org/10.18636/bioneotropical.v3i1.127

[41] R. Sikkema, M. Junginger, P. McFarlane y A. Faaij, "The GHG contribution of the cascaded use of harvested wood products in comparison with the use of wood for energy-A case study on available forest resources in Canada," Environ. Sci. Policy, vol. 31, pp. 96-108, 2013 [En línea]. Disponible en: https://doi.org/10.1016/j.envsci.2013.03.007

[42] A. Thakur, C. E. Canter y A. Kumar, "Life-cycle energy and emission analysis of power generation from forest biomass," Appl. Energy, vol. 128, pp. 246-253, 2014. [En línea]. Disponible en: http://dx.doi.org/10.1016/j.apenergy.2014.04.085

[43] M. Röder, C. Whittaker y P. Thornley, "How certain are greenhouse gas reductions from bioenergy? Life cycle assessment and uncertainty analysis of wood pellet-to-electricity supply chains from forest residues," Biomass and Bioenergy, vol. 79, pp. 50-63, 2015. [En línea]. Disponible en: http://dx.doi.org/10.1016/j.biombioe.2015.03.030

[44] Y. Weldemichael y G. Assefa, "Assessing the energy production and GHG (greenhouse gas) emissions mitigation potential of biomass resources for Alberta," J. Clean. Prod., vol. 112, pp. 4257-4264, 2015. [En línea]. Disponible en: http://dx.doi.org/10.1016/j.jclepro.2015.08.118 
INGE CUC, vol. 13 no. 2, pp 84-94, Julio - Diciembre, 2017

Anexo 1. Las diez especies de mayor IVI en el ecosistema.

\begin{tabular}{|c|c|c|c|c|c|c|c|c|}
\hline $\begin{array}{l}\text { Nombre } \\
\text { científico }\end{array}$ & Familia & $\begin{array}{c}\text { Abundancia } \\
\text { absoluta }\end{array}$ & $\begin{array}{c}\text { Abundancia } \\
\text { relativa }\end{array}$ & $\begin{array}{l}\text { Frecuencia } \\
\text { absoluta }\end{array}$ & $\begin{array}{l}\text { Frecuencia } \\
\text { absoluta }\end{array}$ & $\begin{array}{c}\text { Dominancia } \\
\text { absoluta }\end{array}$ & $\begin{array}{l}\text { Dominancia } \\
\text { relativa }\end{array}$ & IVI \\
\hline $\begin{array}{l}\text { Clathrotropis } \\
\text { brachypetala } \\
\text { (Tul.) } \\
\text { Kleinhoonte }\end{array}$ & Leguminosae & 221 & 5,78 & 12 & 1,22 & 24,01 & 7,34 & 4,78 \\
\hline Inga sp. & Leguminosae & 190 & 4,97 & 10 & 1,02 & 12,82 & 3,92 & 3,30 \\
\hline $\begin{array}{l}\text { Virola sebifera } \\
\text { Aubl. }\end{array}$ & Myristicaceae & 169 & 4,42 & 12 & 1,22 & 10,42 & 3,19 & 2,94 \\
\hline $\begin{array}{l}\text { Pseudolmedia } \\
\text { laevis } \\
\text { (Ruiz \& Pav.) } \\
\text { J.F. Macbr. }\end{array}$ & Moraceae & 167 & 4,37 & 11 & 1,12 & 5,85 & 1,79 & 2,43 \\
\hline $\begin{array}{l}\text { Anacardium } \\
\text { excelsum } \\
\text { (Bertero ex } \\
\text { Kunth) Skeels }\end{array}$ & Anacardiaceae & 13 & 0,34 & 7 & 0,71 & 18,04 & 5,52 & 2,19 \\
\hline Lecythis sp. & Lecythidaceae & 86 & 2,25 & 11 & 1,12 & 9,85 & 3,01 & 2,13 \\
\hline $\begin{array}{l}\text { Hura crepitans } \\
\text { L. }\end{array}$ & Euphorbiaceae & 30 & 0,79 & 9 & 0,92 & 15,27 & 4,67 & 2,12 \\
\hline $\begin{array}{l}\text { Brachycylix } \\
\text { vageleri } \\
\text { (Harms) } \\
\text { Cowan. }\end{array}$ & Leguminosae & 73 & 1,91 & 10 & 1,02 & 10,53 & 3,22 & 2,05 \\
\hline Ceccropia sp. & Leguminosae & 77 & 2.02 & 10 & 1,02 & 9,71 & 2,97 & 2,00 \\
\hline Xylopia sp. & Annonaceae & 117 & 3,06 & 12 & 1,22 & 5,48 & 1,68 & 1,99 \\
\hline
\end{tabular}

Fuente: Autores. 
CATEGORÍA DIAMÉTRICA $(\mathrm{cm})$

\begin{tabular}{|c|c|c|c|c|c|c|c|c|c|c|c|c|c|c|c|c|c|c|c|c|c|c|c|c|c|c|}
\hline CATECORIA & VARIABLE & I(10-20) & $\mathbf{I}(20 \cdot 30)$ & III (30-40) & IV(40-50) & $v(50-60)$ & $\mathrm{v}(160-70)$ & $\mathrm{VI}(\mathbf{7 0 - 8 0 )}$ & VIII(80-90) & $\mathbf{I X}(90-100)$ & $x(100-110)$ & $\mathrm{x}(\mathbf{( 1 1 0 - 1 2 0 )}$ & $\begin{array}{c}\text { xl(1200- } \\
\text { 130) }\end{array}$ & $\begin{array}{c}\mathrm{XIII}(130 . \\
140)\end{array}$ & \begin{tabular}{|c|}
$x y v(140-2$ \\
$150)$
\end{tabular} & $\begin{array}{c}x v(150-\cdot \\
160)\end{array}$ & $\mathrm{x} V(160 \cdot-170)$ & xv॥(770-180) & $\begin{array}{c}\mathrm{XVIII(180.} \\
190)\end{array}$ & $\begin{array}{c}\mathbf{x x}(190 .- \\
200)\end{array}$ & $\mathrm{xx}(200-210)$ & $\operatorname{xx}(210-220)$ & $\begin{array}{c}\mathrm{xxII(2201-} \\
230)\end{array}$ & $\underset{240)}{\operatorname{xxm}(230-}$ & $\begin{array}{c}\mathrm{xxw}_{25040-} \\
250)\end{array}$ & TOTAL \\
\hline \multirow{5}{*}{ 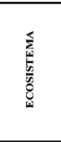 } & \begin{tabular}{|c|} 
Densididd \\
(indo,05 hat
\end{tabular} & 147,08 & 7625 & 38,33 & 25,50 & 11,42 & 9,67 & 4,80 & 2,78 & 2,44 & 2,43 & 1,50 & 1,25 & 1 & 1 & 0 & 0 & 1 & 1 & 1 & 0 & 0 & 0 & a & 1 & 329,45 \\
\hline & 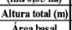 & \begin{tabular}{|l|l|}
$4,035,0$ \\
\end{tabular} & 60.2280 & $8,030.0$ & $6,0,-30.0$ & $10,0.35 .0$ & $11,0.033 .0$ & \begin{tabular}{|l|}
$3,0.35 .0$ \\
\end{tabular} & \begin{tabular}{|c|}
$18,0-40,0$ \\
\end{tabular} & \begin{tabular}{|l|}
$19,0-38.0$ \\
\end{tabular} & $21,032,0$ & $220,(-4), 0$ & $20,0.38 .0$ & 28.0 .33 .0 & \begin{tabular}{|l|l|}
$21.0-31.0$ \\
\end{tabular} & 0 & 0 & 27 & \begin{tabular}{|l|}
$26,0,35.0$ \\
\end{tabular} & 28 & 0 & 0 & 0 & 0 & 28 & 6.040 .0 \\
\hline & \begin{tabular}{|c}
$\begin{array}{c}\text { Areat lasal } \\
(\mathrm{m} 210,015 \mathrm{ha})\end{array}$ \\
\end{tabular} & 263 & 3,53 & 3,46 & 3,86 & 2,60 & 3,10 & 203 & 1,51 & 1,67 & 2,07 & 1,51 & 1,5 & 1,4 & 1,6 & 0 & 0 & 241 & 278 & 2,99 & 0 & 0 & 0 & 0 & 4,75 & 45,39 \\
\hline & 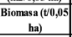 & 15,05 & 23,84 & 27,36 & 33,38 & 24,58 & 30,20 & 21,30 & 15,96 & 18,73 & 23,54 & 19,23 & 17,6 & 18,5 & 18,3 & 0 & 0 & 28,11 & 36,53 & 36,99 & 0 & 0 & 0 & 0 & 57,13 & 465,41 \\
\hline & 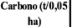 & 7,52 & 11.92 & 13,68 & 16,69 & 1229 & 15,10 & 10,65 & 7.98 & 9.36 & 11,7 & 9.61 & 8.8 & 9,3 & 9,1 & 0 & 0 & 14,05 & 18,26 & 18,04 & 0 & 0 & 0 & 0 & 28,56 & 23271 \\
\hline \multirow{5}{*}{ 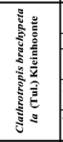 } & \begin{tabular}{|c|c|c|} 
Densidath \\
(inds 0,05 hal
\end{tabular} & 5.45 & 3,36 & 4.27 & 4 & 2.1 & 2 & 1.5 & 0 & 0 & 0 & 0 & 0 & 0 & 0 & 0 & 0 & 0 & 0 & 0 & 0 & 0 & 0 & 0 & 0 & 22,69 \\
\hline & Alturat atsal (m) & \begin{tabular}{|l|l|}
$6,020,0$ \\
\end{tabular} & $6,0,230$ & $9,0,30,0$ & $12,028,0$ & \begin{tabular}{|l|l|}
$15,0.35,0$ \\
\end{tabular} & \begin{tabular}{|l|l|}
$20,0-26,0$ \\
\end{tabular} & \begin{tabular}{|l|l|}
$20,030,0,0$ \\
\end{tabular} & 0 & 0 & 0 & 0 & 0 & 0 & 0 & 0 & 0 & 0 & 0 & 0 & 0 & 0 & 0 & 0 & 0 & $6,0,030,0$ \\
\hline & 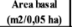 & 0.10 & 0.17 & 0.41 & 0,61 & 0.48 & 0,65 & 0,63 & 0 & 0 & 0 & 0 & 0 & 0 & 0 & 0 & 0 & 0 & 0 & 0 & 0 & 0 & 0 & 0 & 0 & 3,04 \\
\hline & 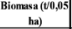 & 0,79 & 1,90 & 5,16 & 8,17 & 6,56 & 8,68 & 8,00 & 0 & 0 & 0 & 0 & 0 & 0 & 0 & 0 & 0 & 0 & 0 & 0 & 0 & 0 & 0 & 0 & 0 & 39,26 \\
\hline & 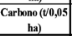 & 0,40 & 0,95 & 2,58 & 4,09 & 3,28 & 4,34 & 4,00 & 0 & 0 & 0 & 0 & 0 & 0 & 0 & 0 & 0 & 0 & 0 & 0 & 0 & 0 & 0 & 0 & 0 & 19,63 \\
\hline \multirow{5}{*}{ 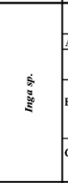 } & 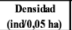 & 7,6 & 7,6 & 275 & 2,4 & 1 & 1,3 & 1 & 1 & 1 & 0 & 0 & 0 & 0 & 0 & 0 & 0 & 0 & 0 & 0 & 0 & 0 & 0 & 0 & 0 & 25,65 \\
\hline & Alturat atsal (min) & 9.01770 & $7,7,-17,0$ & $11,0221,0$ & $16,02,3,0$ & $16,0221,0$ & \begin{tabular}{|l|}
$13,0,22,0$ \\
\end{tabular} & 25 & \begin{tabular}{|l|}
$180-21.0$ \\
\end{tabular} & 28 & 0 & 0 & 0 & 0 & 0 & 0 & 0 & 0 & 0 & 0 & 0 & 0 & 0 & 0 & 0 & $7,0228,0$ \\
\hline & 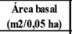 & 0.15 & 0,36 & 0,24 & 0,37 & 0,24 & 0,42 & 0.50 & 0.55 & 0,73 & 0 & 0 & 0 & 0 & 0 & 0 & 0 & 0 & 0 & 0 & 0 & 0 & 0 & 0 & 0 & 3,56 \\
\hline & $\begin{array}{c}\text { Bionasas }(v v 0,05 \\
\text { hay })\end{array}$ & 1,28 & 3,89 & 299 & 5,02 & 3,28 & 5,60 & 6,10 & 6,61 & 7,98 & 0 & 0 & 0 & 0 & 0 & 0 & 0 & 0 & 0 & 0 & 0 & 0 & 0 & 0 & 0 & 42,77 \\
\hline & 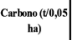 & 0.64 & 1.55 & 1.50 & 2.51 & 1.64 & 2.80 & 3.05 & 3,00 & 3.99 & 0 & 0 & 0 & 0 & 0 & 0 & 0 & 0 & 0 & 0 & 0 & 0 & 0 & 0 & 0 & 21.38 \\
\hline \multirow{5}{*}{ 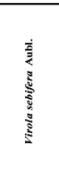 } & 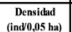 & 7 & 5,1 & 2,37 & 2,16 & 1,28 & 1 & 0 & 0 & 0 & 0 & 0 & 0 & 0 & 0 & 0 & 0 & 0 & 0 & 0 & 0 & 0 & 0 & 0 & 0 & 18,91 \\
\hline & Alturat bxal (mi) & \begin{tabular}{|l|l|}
$7,018,0$ \\
\end{tabular} & $80-2200$ & $12,0.30 .0$ & $15,0.28,0$ & \begin{tabular}{|l|l|}
$19,030,0$ \\
\end{tabular} & \begin{tabular}{|l|}
$18,0,32.0$ \\
\end{tabular} & 0 & 0 & 0 & 0 & 0 & 0 & 0 & 0 & 0 & 0 & 0 & 0 & 0 & 0 & 0 & 0 & 0 & 0 & $7.0,32.0$ \\
\hline & 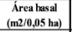 & 0,12 & 0,23 & 0,19 & 0,33 & 0,29 & 0,31 & 0 & 0 & 0 & 0 & 0 & 0 & 0 & 0 & 0 & 0 & 0 & 0 & 0 & 0 & 0 & 0 & 0 & 0 & 1,46 \\
\hline & $\begin{array}{c}\text { Bionasas }(v 0,05 \\
\text { ha })\end{array}$ & 0.95 & 2,12 & 2,35 & 4,44 & 4,00 & 4,13 & 0 & 0 & 0 & 0 & 0 & 0 & 0 & 0 & 0 & 0 & 0 & ${ }^{0}$ & 0 & ${ }^{0}$ & 0 & ${ }^{0}$ & 0 & 0 & 18,28 \\
\hline & $\begin{array}{c}\text { Carkmeno(tv0,05 } \\
\text { ha) }\end{array}$ & 0,48 & 1,21 & 1,17 & 2,22 & 2,00 & 2,06 & 0 & 0 & 0 & 0 & 0 & 0 & ${ }^{0}$ & 0 & 0 & 0 & 0 & 0 & 0 & 0 & ${ }^{0}$ & 0 & 0 & 0 & 9,14 \\
\hline \multirow{5}{*}{ 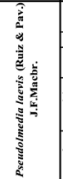 } & \begin{tabular}{|c|c|} 
Densidad \\
Contusus
\end{tabular} & 10,090509091 & 2,6363636066 & 1.857142357 & 1 & 1 & 1 & 1 & 0 & 0 & 0 & 0 & 0 & 0 & 0 & 0 & 0 & 0 & 0 & 0 & 0 & 0 & 0 & 0 & 0 & 19,40 \\
\hline & Alturat total (m) & \begin{tabular}{|l|l|}
$7,020,0$ \\
\end{tabular} & $11,026,9$ & $14,0.20,0$ & $19,021,0$ & 20 & 19 & 25 & 0 & 0 & 0 & 0 & 0 & 0 & 0 & 0 & 0 & 0 & 0 & 0 & 0 & 0 & 0 & 0 & 0 & $7,025,0$ \\
\hline & 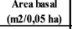 & 0.18 & 0,12 & 0.17 & 0,15 & 0,25 & 0.35 & 0,40 & 0 & 0 & 0 & 0 & 0 & 0 & 0 & 0 & 0 & 0 & 0 & 0 & 0 & 0 & 0 & 0 & 0 & 1,62 \\
\hline & $\begin{array}{c}\text { Bionasas (tvo,05 } \\
\text { has) }\end{array}$ & 1,44 & 1,35 & 220 & 1,96 & 3,41 & 4,64 & 5,12 & 0 & 0 & 0 & 0 & 0 & 0 & 0 & 0 & 0 & 0 & 0 & 0 & 0 & 0 & 0 & 0 & 0 & 20,13 \\
\hline & 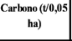 & 0.72 & 0.68 & 1.10 & 0.98 & 1,71 & 2.32 & 2.56 & 0 & 0 & 0 & 0 & 0 & 0 & 0 & 0 & 0 & 0 & 0 & 0 & 0 & 0 & ${ }^{0}$ & 0 & 0 & 10.07 \\
\hline \multirow{5}{*}{ 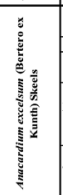 } & 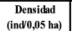 & 1,5 & 0 & 1 & 0 & 1 & 0 & 0 & 0 & 0 & 1 & 1 & 1 & 1 & 0 & 0 & 0 & 0 & 1 & 1 & 0 & 0 & 0 & 0 & 1 & 10,5 \\
\hline & Alturatat total (min & \begin{tabular}{|l|l}
$11,02-26,0$ \\
\end{tabular} & 0 & 24 & 0 & 22 & 0 & 0 & 0 & 0 & 25 & 30 & 22 & 32 & 0 & 0 & 0 & 0 & 35 & 28 & 0 & 0 & 0 & 0 & 28 & $11,0-35.0$ \\
\hline & 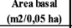 & 0.03 & 0 & 0.11 & 0 & 0,27 & 0 & 0 & 0 & 0 & 0.83 & 0.96 & 1.19 & 1.33 & 0 & 0 & 0 & 0 & 2,78 & 2.99 & 0 & 0 & 0 & 0 & 4,75 & 15,24 \\
\hline & $\begin{array}{c}\text { Biomasas }(v 10,05 \\
\text { ha) }\end{array}$ & 0,20 & 0 & 1,49 & 0 & 3,70 & 0 & 0 & 0 & 0 & 8.56 & 9,22 & 9,22 & 10,64 & 0 & 0 & 0 & 0 & 12,38 & 12,39 & 0 & 0 & 0 & 0 & 11,71 & 79,52 \\
\hline & 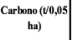 & 0,10 & 0 & 0,74 & 0 & 1,85 & 0 & 0 & 0 & 0 & 428 & 4,61 & 4,61 & 5,32 & 0 & 0 & 0 & 0 & 6,19 & 6,20 & 0 & 0 & 0 & 0 & 5,85 & 39,76 \\
\hline \multirow{5}{*}{ 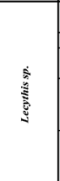 } & \begin{tabular}{|c}
$\begin{array}{c}\text { Densidad } \\
(\text { indo,05 ha })\end{array}$ \\
\end{tabular} & 4,286 & 2,444 & 1,167 & 2 & 1,5 & 1 & 1 & 0 & 1 & 1 & 1 & 0 & 0 & 0 & 0 & 0 & 0 & 0 & 0 & ${ }_{0}$ & 0 & 0 & 0 & 0 & 16,40 \\
\hline & Alturat atal (ini) & $7,018.0$ & $10,025.0$ & $12,0-21.0$ & $6,0,22,0$ & $16,024,0$ & $2,026,0$ & \begin{tabular}{|l|l|}
$19,0-21.0$ \\
\end{tabular} & 0 & 27 & 25 & 28 & 0 & 0 & 0 & 0 & 0 & 0 & 0 & 0 & 0 & 0 & 0 & 0 & 0 & $7,028,0$ \\
\hline & 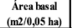 & 0,08 & 0,11 & 0.11 & 0,32 & 0,33 & 0,30 & 0,41 & 0 & 0,64 & 0,79 & 0,95 & 0 & 0 & 0 & 0 & 0 & 0 & 0 & 0 & 0 & 0 & 0 & 0 & 0 & 4,04 \\
\hline & $\begin{array}{c}\text { Biomasas }(v 0,05 \\
\text { ha) }\end{array}$ & 0,61 & 1,20 & 1.36 & 4,35 & 4,49 & 4,05 & 5,26 & 0 & 7.26 & 8.33 & 9.18 & 0 & 0 & 0 & 0 & 0 & 0 & 0 & 0 & 0 & 0 & 0 & 0 & 0 & 46,10 \\
\hline & $\begin{array}{c}\text { Carlomo }(v 0,05 \\
\text { ha) }\end{array}$ & 0,31 & 0,60 & 0,68 & 2,18 & 2,25 & 2,03 & 2,63 & 0 & 3,27 & 4,16 & 4,59 & 0 & 0 & 0 & 0 & 0 & 0 & 0 & 0 & 0 & 0 & ${ }^{0}$ & 0 & 0 & 22,69 \\
\hline \multirow{5}{*}{ 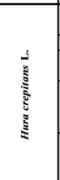 } & \begin{tabular}{|c|c|} 
Densidad \\
(inds 0,05 hal
\end{tabular} & 2 & 1 & 1 & 1 & 1 & 1 & 1 & 2 & 1 & 1 & 1.5 & 0 & 0 & 1.25 & 0 & 0 & 0 & 0 & 0 & 0 & 0 & 0 & 0 & 0 & 14,75 \\
\hline & Alturat atsal (m) & \begin{tabular}{|l|l|}
$8,024,0$ \\
\end{tabular} & $14,016,0,0$ & 33 & \begin{tabular}{ll|}
$42,048,5,5$ \\
\end{tabular} & 23 & \begin{tabular}{|l|}
$19,026,0$ \\
\end{tabular} & \begin{tabular}{|l|}
$220,25,0$ \\
\end{tabular} & \begin{tabular}{|l|}
$21,0-025,0$ \\
\end{tabular} & 20 & 27 & \begin{tabular}{|l|}
$22,0,(-4), 0$ \\
\end{tabular} & 0 & 0 & $21,031,0$ & 0 & 0 & 0 & 0 & 0 & 0 & 0 & 0 & 0 & 0 & $8,040,0$ \\
\hline & 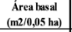 & 0,03 & 0,94 & 0,09 & 0,16 & 0,20 & 0,34 & 0.41 & 1,14 & 0,66 & 0,83 & 1,53 & 0,00 & 0,000 & 1,86 & 0 & 0 & 0 & 0 & 0 & 0 & 0 & 0 & 0 & 0 & 7,30 \\
\hline & $\begin{array}{c}\text { Biomanas }(v 0,05 \\
\text { ha })\end{array}$ & 0.25 & 0,44 & 1,07 & 2,19 & 279 & 4,47 & 5,27 & 13,4 & 7,48 & 8,56 & 14,26 & 0,00 & 0,00 & 13,65 & 0 & 0 & 0 & 0 & 0 & ${ }^{0}$ & 0 & ${ }^{\circ}$ & o & ${ }^{\circ}$ & 73,88 \\
\hline & 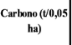 & 0.13 & 0.22 & 0.53 & 1.10 & 1.40 & 2,23 & 2.64 & 6,72 & 3.74 & 428 & 7.13 & 0.00 & 0.00 & 6.83 & 0 & 0 & 0 & 0 & 0 & 0 & 0 & ${ }^{0}$ & 0 & 0 & 36,94 \\
\hline 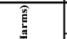 & 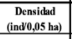 & 3,2 & 2,25 & 2 & 2,33 & 1,33 & 1,75 & 1,5 & 1 & 1 & 0 & 0 & 0 & 0 & 0 & 0 & 0 & 0 & 0 & 0 & ${ }^{0}$ & 0 & 0 & 0 & 0 & 16,36 \\
\hline & Alturat tral (min) & $\begin{array}{l}8,025,0 \\
\end{array}$ & $12,022,0$ & $120,021,0$ & $17,0,28,0$ & $16,0,26,0$ & $20,028,0$ & 211,0-24,0 & $24,0-27,0$ & $21,0,30,0$ & 0 & 0 & 0 & 0 & 0 & 0 & 0 & 0 & 0 & 0 & 0 & 0 & 0 & 0 & 0 & $8,0,30,0$ \\
\hline ) & 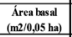 & 0.06 & 0,11 & 0,18 & 0,35 & 0,33 & 0,56 & $0, \infty$ & $0, \infty 0$ & $0,0,0$ & 0 & 0 & 0 & 0 & 0 & 0 & 0 & 0 & 0 & 0 & 0 & 0 & ${ }^{\circ}$ & 0 & 0 & 3,49 \\
\hline & $\begin{array}{c}\text { Binmasa }(v 0,0,5 \\
\text { hay), }\end{array}$ & 0,54 & 1,17 & 2313 & 4,71 & 4,46 & 7,46 & 7,73 & 7,00 & 7,73 & 0 & 0 & 0 & 0 & 0 & 0 & 0 & 0 & 0 & 0 & 0 & 0 & 0 & 0 & 0 & 43,11 \\
\hline 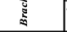 & 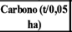 & 0.27 & 0.58 & 1.15 & 2,35 & 2,23 & 3.73 & 3.87 & 3,50 & 3.87 & 0 & 0 & 0 & 0 & 0 & 0 & 0 & 0 & 0 & 0 & 0 & 0 & 0 & 0 & 0 & 21,56 \\
\hline & \begin{tabular}{|c|c|} 
Densidad \\
(inde, 05 ha)
\end{tabular} & 3.44 & 2,66 & 1,75 & 1,4 & 1,2 & 1 & 1,5 & 1 & 2 & 1 & 1 & 0 & 0 & 0 & 0 & 0 & 0 & 0 & 0 & 0 & 0 & 0 & 0 & 0 & 17,95 \\
\hline & 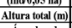 & $5,0255,0$ & \begin{tabular}{|l|}
$11,0221,0$ \\
\end{tabular} & $8,021.0$ & $9,0.26,0$ & $14,028,0$ & $18,025.0$ & \begin{tabular}{|l|l}
$13,0.27 .0$ \\
\end{tabular} & 21 & \begin{tabular}{|l|}
$21,0-23,0$ \\
\end{tabular} & 25 & 35 & 0 & 0 & 0 & 0 & 0 & 0 & 0 & 0 & 0 & 0 & 0 & 0 & 0 & $5,0,35,0$ \\
\hline 8 & \begin{tabular}{|c|}
$\begin{array}{c}\text { Areat basal } \\
(\mathrm{m} / 2 / 0,05 \mathrm{ha})\end{array}$ \\
\end{tabular} & 0,07 & 0,13 & 0.15 & 0,21 & 0,26 & 0,28 & 0,59 & 0,57 & 1.29 & 0,85 & 0.95 & 0 & 0 & 0 & 0 & 0 & 0 & 0 & 0 & 0 & 0 & 0 & 0 & 0 & 5,34 \\
\hline है & 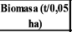 & 0.59 & 1,39 & 1,82 & 2,83 & 3,52 & 3,82 & 7,62 & 6,72 & 14,69 & 8,65 & 9.18 & 0 & 0 & 0 & 0 & 0 & 0 & 0 & 0 & 0 & 0 & 0 & 0 & 0 & 60,83 \\
\hline & 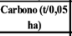 & 0,29 & 0,70 & 0,91 & 1,41 & 1,76 & 1,91 & 3,81 & 3,36 & 7,34 & 4,33 & 4,59 & 0 & 0 & 0 & 0 & 0 & 0 & 0 & 0 & 0 & 0 & 0 & 0 & 0 & 30,42 \\
\hline & 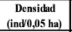 & 5,44 & 3,22 & 243 & 1,29 & 0 & 1 & 0 & 0 & 0 & 0 & 0 & 0 & 0 & 0 & 0 & 0 & 0 & 0 & 0 & 0 & 0 & 0 & 0 & 0 & 13,4742857 \\
\hline & & $6,022,0$ & \begin{tabular}{|l|}
$11,0,22,0$ \\
\end{tabular} & $13,0.22,0$ & $19,0.50,0$ & 0 & 25 & 0 & 0 & 0 & 0 & 0 & 0 & 0 & 0 & 0 & 0 & 0 & 0 & 0 & 0 & 0 & 0 & 0 & 0 & \begin{tabular}{|c|}
$6,030,0$ \\
\end{tabular} \\
\hline 8 & 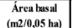 & 0.10 & 0.14 & 0.20 & 0.19 & 0 & 0.37 & 0 & 0 & 0 & 0 & 0 & 0 & 0 & 0 & 0 & 0 & 0 & 0 & 0 & 0 & 0 & 0 & 0 & 0 & 1.01 \\
\hline 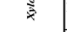 & $\begin{array}{l}\text { Biomass (v010,05 } \\
\text { hay) }\end{array}$ & 0.78 & 1.54 & 2.50 & 2,02 & 0 & 4.88 & 0 & 0 & 0 & 0 & 0 & 0 & 0 & 0 & 0 & 0 & 0 & 0 & 0 & 0 & 0 & 0 & 0 & 0 & 12,32 \\
\hline & 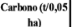 & 0.39 & 0,77 & 1,25 & 1,31 & 0 & 2,4 & 0 & 0 & 0 & 0 & 0 & 0 & 0 & 0 & 0 & 0 & 0 & 0 & 0 & 0 & 0 & 0 & 0 & 0 & 6,16 \\
\hline
\end{tabular}

Fuente: Autores. 